\title{
ESENVOLVIMENTO DE UM MODELO DINÂMICO E ADAPTATIVO COMO FERRAMENTA PARA A ORIENTAÇÃO ESTRATÉGICA EM ORGANIZAÇÕES DO SETOR AEROESPACIAL
}

Recebido: 07/11/2019
Aprovado: $10 / 02 / 2020$

\author{
1Dinah Eluze Sales Leite \\ 2Milton de Freitas Chagas Júnior
}

\section{Resumo}

Objetivo: O artigo propõe um modelo estratégico, dinâmico e adaptativo com o objetivo de melhorar a captura e o aproveitamento de oportunidades na seleção do portfólio de tecnologias em ambientes complexos e incertos.

Método: A metodologia definida nesta pesquisa é o estudo de casos múltiplos na abordagem Design Science. Através do desenvolvimento e da aplicação do modelo proposto, busca-se gerar conhecimento científico sobre a seleção de tecnologias em organizações do setor aeroespacial. O estudo também considera uma forte combinação das lógicas indutiva e abdutiva, para lidar com a incerteza inerente ao ambiente de P\&D.

Originalidade/Relevância: Do ponto de vista teórico, pode-se destacar, como principais lacunas da estratégia tecnológica no setor aeroespacial, as tomadas de decisão sob incerteza, a falta de integração entre as áreas nas tomadas de decisão e as limitações no horizonte de tempo do desenvolvimento estratégico.

Resultados: Os principais resultados esperados, com a aplicação do modelo são: (1) melhorar a captura e o aproveitamento de oportunidades, (2) melhorar a vantagem competitiva da organização e (3) contribuir com uma estratégia tecnológica mais flexível em longo prazo. Os casos explorados permitiram o detalhamento dos impactos das tomadas de decisão sobre o desenvolvimento estratégico e do domínio das tecnologias na definição das rotas tecnológicas.

Contribuições teóricas: A pesquisa contribui, do ponto de vista teórico, com o modelo de capacidades dinâmicas, ao incluir o seizing ao generative sensing.

Contribuições para gestão: Do ponto de vista prático, o desenvolvimento do modelo contribui com a gestão estratégica das organizações aeroespaciais, no sentido de tornarem-nas mais robustas, ágeis e flexíveis.

Palavras-chave: Estratégia tecnológica. Capacidades dinâmicas. Incerteza. Ambidestria. Tomada de decisão.

\section{Como Citar:}

Sales Leite, D., \& Chagas Jr, M. (2020). Desenvolvimento de um Modelo Dinâmico e Adaptativo como Ferramenta para a Orientação Estratégica em Organizações do Setor Aeroespacial. Future Studies Research Journal: Trends and Strategies [FSRJ], 12(2), 242-263. doi:https://doi.org/10.24023/FutureJournal/2175$\underline{5825 / 2020 . v 12 i 2.472}$

\footnotetext{
1 Instituto Nacional de Pesquisas Espaciais - INPE, São José dos Campos, (Brasil). E-mail: salesleite@gmail.com Orcid id: https://orcid.org/0000-0001-7044-5994

2 Instituto Nacional de Pesquisas Espaciais - INPE, São José dos Campos, Brasil). E-mail: milton.chagas@inpe.br Orcid id: https://orcid.org/0000-0002-5409-0457
} 


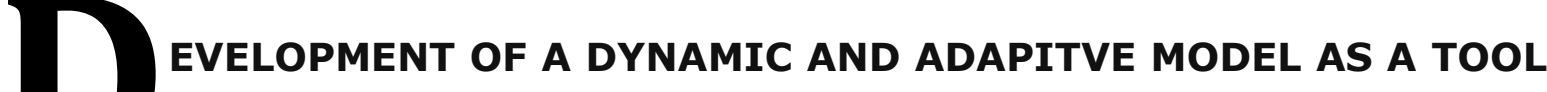 FOR STRATEGIC GUIDANCE IN AEROSPACE ORGANIZATIONS
}

\begin{abstract}
Objective: The article proposes a strategic model, dynamic and adaptive, aiming to improve the capture and use of opportunities in the selection of the technology portfolio in complex and uncertain environments.

Method: The methodology defined in this research is the study of multiple cases in the Design Science approach. We seek to generate scientific knowledge about the selection of technologies in organizations in the aerospace sector through the development and application of the proposed model. The study also considers a combination of inductive and abductive logic to deal with the inherent uncertainty in the R\&D environment.
\end{abstract}

Originality/Relevance: From a theoretical point of view, the main gaps in technological strategy in the aerospace sector can be highlighted: decision making under uncertainty, the lack of integration between areas in decision making, and limitations in the time horizon of strategic development.

Results: The main expected results, with the application of the model, are: (1) improve the capture, and use of opportunities, (2) improve the competitive advantage of the organization, and (3) contribute to a more flexible technology strategy in the long term. The cases explored allowed the detailing of the impacts of decision making on strategic development and the technologies domain in the definition of technological routes.

Theoretical contributions: From a theoretical point of view, the research contributes to the dynamic capabilities model, including seizing to generative sensing.

Management contributions: From a practical point of view, the development of the model contributes to aerospace organizations' strategic management to make them more robust, agile, and flexible.

Keywords: Technological strategy. Dynamic capabilities. Uncertainty. Ambidexterity. Decision making.

\section{How to cite the article:}

Sales Leite, D., \& Chagas Junior, M. (2020). Development of a Dynamic and Adaptive Model as a Tool for Strategic Guidance in Aerospace Organizations. Future Studies Research Journal: Trends and Strategies, 12(2), 242-263. doi:https://doi.org/10.24023/FutureJournal/2175-5825/2020.v12i2.472 


\section{Introdução}

As dinâmicas estratégicas em organizações do setor aeroespacial envolvem grande complexidade e altos níveis de incertezas. Essas organizações realizam múltiplos investimentos em projetos de pesquisa e desenvolvimento ( $P \& D)$, mas têm dificuldades em prever retornos, econômicos e científicos. A estratégia tecnológica, fortemente relacionada ao retorno dos investimentos, pode impactar o crescimento e a sustentabilidade das organizações.

As pesquisas relacionadas aos fatores estratégicos envolvem a visão baseada em recursos (Wernerfelt, 1984) e a compreensão em relação ao sucesso dos investimentos, crescimento e perpetuidade da organização. Prahalad e Hamel (1990), ao abordarem o conceito de competências essenciais, contribuíram com elementos centrais de uma estratégia organizacional. A literatura inclui as estratégias baseadas em recursos, que ganharam destaque principalmente nos trabalhos de Penrose (1959), Wernerfelt (1984), Grant (1991) e Barney (1991). Mais tarde, com a evolução das pesquisas econômico administrativas, o conceito de capacidades dinâmicas foi destacado nos trabalhos de Teece e Pisano (1994), e Teece, Pisano e Shuen (1997). Devido à importância da adaptabilidade das organizações em ambientes de grandes transformações, o conceito continuou sendo atualizado, (Teece, 2007, Augier \& Teece, 2008, Teece, Peteraf, \& Leih, 2016, Pisano, 2016, Teece, 2018).

Problemas relacionados à falta de agilidade são muito comuns nas organizações de grande porte. Neste contexto, os tomadores de decisão perceberam a necessidade de combater a rigidez da estratégia organizacional para assegurarem a flexibilização estratégica e a competitividade, frente às mudanças descontínuas (Levinthal, 1992; Teece, Pisano \& Shuen, 1997; Eisenhardt \& Martin 2000; Helfat \& Peteraf, 2003; Rohrbeck, 2010). Esta análise, que inclui a flexibilidade estratégica e a adaptação às mudanças, mostra que a mortalidade de grandes organizações pode ser explicada por sua capacidade de identificar, preparar e responder às mudanças descontínuas (Brown \& Eisenhardt, 1997; Winter, 2004; Rohrbeck, 2010, 2014; Drucker, 2011; Rohrbeck \& Schwarz, 2013; Schwarz, Rohrbeck, \& Wach, 2019). As contribuições desta pesquisa com a prática incluem (1) o desenvolvimento de um modelo, dinâmico e adaptativo, baseado nas capacidades dinâmicas e (2) a identificação das melhores práticas em relação aos elementos considerados no modelo. Estes objetivos buscam melhorar a sustentabilidade e a competitividade das organizações do setor aeroespacial frente às mudanças do ambiente. Em relação à teoria, as contribuições esperadas incluem (1) a análise das lacunas metodológicas de pesquisas anteriores e a (2) expansão do conceito "generative sensing" para "generative sensing e seizing". 
Busca-se melhorar a forma de lidar com a incerteza, aumentando o grau de confiança dos tomadores de decisão no desenvolvimento da estratégia tecnológica.

\section{Revisão de Literatura}

\section{Capacidades dinâmicas, Incertezas e Tomadas de Decisão}

As capacidades dinâmicas fundamentam o desenvolvimento estratégico das organizações (Teece \& Pisano, 1994, Teece, Pisano, \& Shuen, 1997, Teece, 2007) assegurando a agilidade necessária para lidar com incerteza (Teece, Peteraf, \& Leih, 2016). Estas capacidades constituem uma abordagem estratégica que explica como as organizações se adaptam e reconfiguram suas capacidades dinâmicas e operacionais para responderem e se anteciparem às mudanças (Teece, Peteraf, \& Leih, 2016), aproveitando as oportunidades e mantendo a vantagem competitiva em um mercado caracterizado por contínuas flutuações (Eisenhardt \& Martin, 2000).

O segredo da vantagem competitiva de algumas organizações de sucesso reside em uma estratégia que favorece a captura de oportunidades, nas quais o tomador de decisão explora mercados complexos, avalia oportunidades, cria e testa hipóteses e muda as decisões de acordo com as circunstâncias, dando flexibilidade ao processo (Eisenhardt \& Sull, 2001, Dong, Garbuio, \& Lovallo, 2016).

As capacidades dinâmicas podem ser observadas em ambientes de alta tecnologia nos quais a busca por informações suporta rápidas mudanças. Estas condições exigem que as organizações desenvolvam mecanismos dinâmicos para assegurarem vantagem competitiva e crescimento econômico. O artigo detalha esse ambiente onde as decisões precisam ser tomadas, com ou sem informações suficientes.

Figura 1: Capacidades dinâmicas relacionadas às métricas de TRL

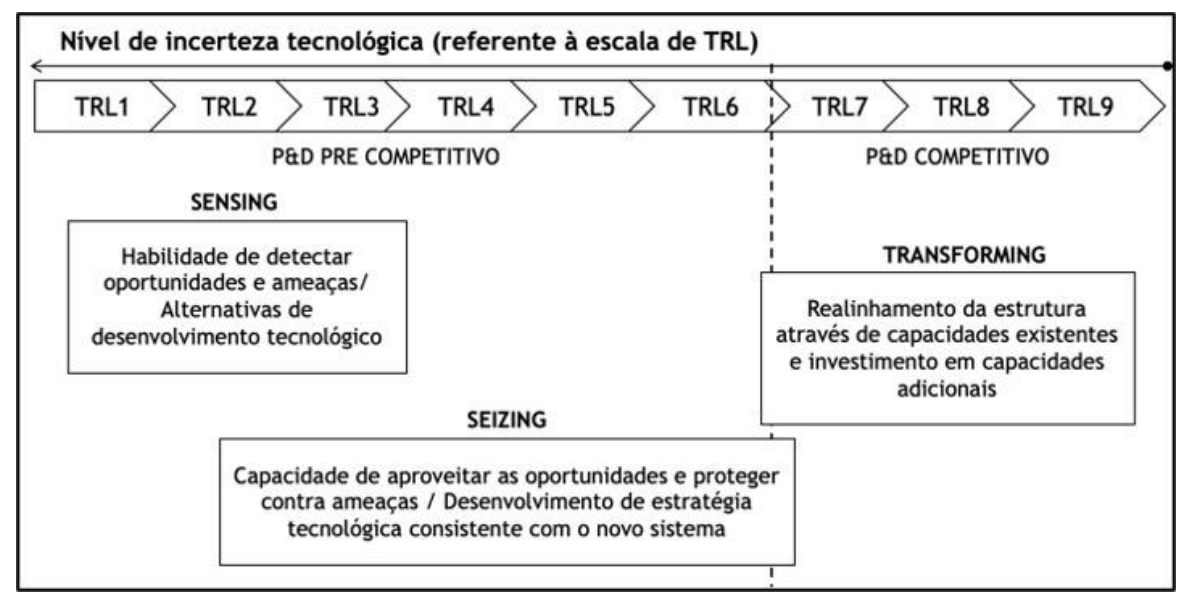

Fonte: Leite \& Chagas Jr., 2019 
Teece (2007) dividiu as capacidades dinâmicas em três elementos principais: "sensing", "seizing" e "transforming". Este estudo considera o "sensing" - capacidade de detectar oportunidades e ameaças, e o "seizing" - capacidade de aproveitar as oportunidades e proteger contra as ameaças do mercado. Esta estrutura, que deriva da visão baseada em recursos (Penrose, 1959) explica como as organizações moldam e reconfiguram seus recursos estratégicos para manterem vantagem competitiva.

A combinação das capacidades dinâmicas gera não apenas uma estratégia tecnológica, mas um modelo de negócios, permitindo a transformação organizacional (Figura 1). Esse modelo pode trazer vantagens para a organização, detectando alternativas de desenvolvimento ("sensing") e a capacidade de se construir uma estratégia tecnológica coerente com o novo sistema ("seizing"). Em sistemas complexos de organizações de base tecnológica, o desenvolvimento de algumas tecnologias precisa avançar, portanto, a estratégia tecnológica precisa ser independente do programa.

\section{Nível de maturidade tecnológica (TRL - Technology Readiness Level)}

O gerenciamento dos investimentos em tecnologia é fundamental para o sucesso dos projetos e programas e se baseiam nos argumentos de que podem reduzir as incertezas relacionadas ao desempenho, tempo e custo (Mankins, 2009a). Se a pesquisa e o desenvolvimento tecnológico tiverem implementação antecipada, os sistemas compostos por essas tecnologias podem resultar em custos excessivos, atrasos e a erosão dos objetivos iniciais de desempenho.

Os custos associados ao nível 1 de TRL podem variar de uma pequena fração da aplicação da tecnologia a um valor superior ao próprio sistema. No TRL 2 os custos representam uma pequena fração da tecnologia no sistema. Nos níveis 3 e 4 , os custos costumam ser baixos a moderados. No nível 5 os custos geralmente são moderados a altos. Para o TRL 6, os custos são elevados e incluem demonstradores tecnológicos (Mankins, 2009b).

Figura 2: Modelo TRRA

\begin{tabular}{|c|c|c|c|c|}
\hline & \multicolumn{4}{|c|}{$\begin{array}{l}\text { grau de maturidade tecnológica, dificuldade de desenvolvimento e } \\
\text { importância da tecnologia para o sucesso do programa }\end{array}$} \\
\hline & TRL3 & TRL4 & TRL5 & TRL6 \\
\hline $\begin{array}{l}\text { Parâmetros de } \\
\text { desempenho }\end{array}$ & iniciais & melhorados & melhor & objetivos alcançados \\
\hline Fases de PधD & inicial & intermediária & $\begin{array}{l}\text { definiçāo da } \\
\text { arquitetura de } \\
\text { sistemas }\end{array}$ & $\begin{array}{l}\text { decisão final no } \\
\text { desenvolvimento do } \\
\text { sistema }\end{array}$ \\
\hline $\begin{array}{l}\text { INCERTEZA } \\
\text { CONCEITUALL }\end{array}$ & ALTA & \multicolumn{2}{|c|}{ MÉDIA } & BAIXA \\
\hline & \multicolumn{2}{|c|}{$\downarrow$} & $\downarrow$ & \\
\hline & $\begin{array}{l}\text { Teste de componentes } \\
\text { individuais }\end{array}$ & $\begin{array}{l}\text { Sistemas de alta } \\
\text { fidelidade }\end{array}$ & $\begin{array}{l}\text { Demonstração em } \\
\text { laboratório }\end{array}$ & $\begin{array}{l}\text { Demonstração em } \\
\text { ambiente real }\end{array}$ \\
\hline & \multicolumn{4}{|c|}{ MODELAGEM E SIMULAÇÃO } \\
\hline
\end{tabular}

Fonte: adaptado de Mankins, 2009a 
O modelo TRRA ("Technology Readiness and Risk Assessment") inclui desenvolvimento tecnológico e os elementos de análise de risco em cada nível de TRL (Mankins, 2009a; Azizian, Sarkani \& Mazzuchi, 2009). O modelo consolida TRL, Grau de Dificuldade em P\&D (P\&D3), e Valor da Necessidade Tecnológica (TNV), que avalia a importância do desenvolvimento de uma tecnologia para o sucesso do programa. Os investimentos em $P \& D$ podem melhorar os parâmetros de desempenho, as aplicações do sistema e a redução de riscos para as atividades subsequentes, neste estudo abordados como incertezas (Leite \& Chagas Jr., 2019). O mais crítico da tomada de decisão é avaliar se as tecnologias necessárias para o sistema atingiram coletivamente o ponto de maturidade, risco, incerteza e desempenho necessários para continuar o desenvolvimento do sistema (Figura 2), que pode impactar o sucesso ou o fracasso dos programas (Mankins, 2009a). O sucesso de um programa de longo prazo depende de metodologias que contribuam para a identificação de necessidades e oportunidades em desenvolvimentos futuros (Mankins, 2002).

\section{Planejamento de cenários}

O planejamento de cenários constitui uma metodologia essencial para a análise prospectiva, permitindo o estudo de futuros possíveis e desejáveis e a estruturação da trajetória entre as situações presente e futura (Godet, 1983). Trata-se de um método orientado para o futuro (Schwartz, 2008), que permite o uso sistemático de "insights" na análise dos impactos das incertezas (Van der Heijden, Bradfield, Burt, Cairns, \& Wright, 2002).

A relevância do planejamento de cenários está na construção de futuros plausíveis e geração de estratégias que possam contribuir para a captura de oportunidades, proteção contra as ameaças e minimização das incertezas (Ramírez \& Selin, 2014). Desta forma, o desenvolvimento de cenários pode facilitar a exploração de alternativas futuras (Schoemaker, 1995), como ferramenta de apoio ao processo decisório (Schwartz, 1991).

Van der Heijden (2005) inclui as incertezas inerentes ao ambiente organizacional como elemento central de um planejamento estratégico eficaz. O planejamento de cenários está além do desenvolvimento estratégico e inclui antecipação, "sensemaking" e aprendizado organizacional. De acordo com Courtney, Kirkland e Viguerie (1997), as organizações devem desenvolver de cenários futuros para se adaptarem às incertezas.

Para lidar com as incertezas, Kwakkel e Pruit (2013) se baseiam na exploração de futuros plausíveis, estabelecendo a relação da modelagem exploratória com o planejamento de cenários para apoiar a tomada de decisão sob incerteza. As análises 
de futuro têm impacto no planejamento de longo prazo e estimulam o tomador de decisão a agir de maneira diferente de suas referências passadas, ou permanecer no universo da especulação (Wack, 1985).

\section{Metodologia}

O método definido para este estudo de casos múltiplos é a abordagem "Design Science". Através do desenvolvimento e da aplicação de um artefato, busca-se gerar conhecimento científico sobre a seleção de tecnologias em organizações da indústria aeroespacial. O estudo considera uma forte combinação das lógicas indutiva e abdutiva, para lidar com a incerteza no ambiente de P\&D (Figura 3). Esses fatores são essenciais para a melhor compreensão dos processos de tomada de decisões a partir de evidências empíricas.

A indução é definida como o raciocínio que resulta da inferência e que leva à verdade em longo prazo, e a abdução representa um processo de inferência usado para explicar evidências, explorar dados, identificar padrões, sugerir hipóteses e permitir novas descobertas (Peirce, 1974).

Figura 3: Dedução, Indução e Abdução

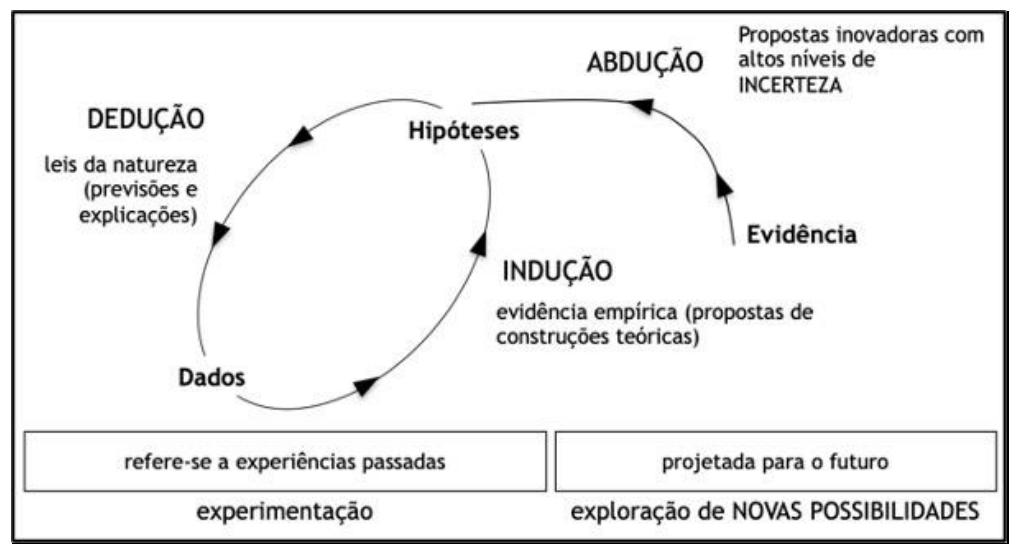

Fonte: adaptado de Peirce, 1998

Peirce definiu a abdução como um raciocínio construído a partir de um estado futuro desconhecido. Os raciocínios dedutivo e indutivo não geram ideias, cuja origem está na abdução (Peirce, 1974, 1998). O uso do raciocínio abdutivo (Peirce, 1974) na metodologia "Design Science" pode melhorar a capacidade de desenvolver novas e diferentes soluções (Hevner, March, Park, \& Ram, 2004). Essa solução, que pode passar por hipóteses, é utilizada para desenvolver, testar e implementar um artefato, representado pelo modelo proposto. 


\section{Estudo de casos múltiplos}

Os estudos de caso fragmentam os casos em múltiplas dimensões, permitindo o reconhecimento de padrões e relações entre eles (Eisenhardt, 1989), incluindo desafios que podem ser mitigados através de projetos de pesquisa bem estruturados (Eisenhardt \& Graebner, 2007). Os benefícios do estudo de casos múltiplos incluem a melhor compreensão dos eventos, testes da teoria existente e o desenvolvimento de uma nova abordagem. A seleção dos casos é extremamente importante para o desenvolvimento de uma teoria emergente (Einsenhardt, 1989).

\section{Design Science (DS)}

Segundo De Sordi, Azevedo, Meirelles e Campanario (2013), a abordagem DS teve início na década de sessenta, 1963, com os trabalhos de Fuller e McHale. Em 1968, com "The Sciences of the Artificial", a metodologia foi amplamente divulgada (Simon, 1996). Simon definiu a solução de problemas pela ciência do artificial e destacou que os artefatos construídos a partir das técnicas de pesquisa científica, são essenciais para a tomada de decisão.

Hevner, March, Park e Ram (2004) definiram a abordagem "Design Science" como uma ciência inerente ao processo de resolução de problemas, no qual o princípio fundamental, de onde derivam sete diretrizes, contempla a compreensão de um problema e sua solução, a partir da construção e aplicação de um artefato. As diretrizes são:

- Projeto de um artefato: a pesquisa em DS exige a criação de um artefato inovador e útil;

- Relevância do problema: a pesquisa deve ser direcionada para o domínio de problema;

- Avaliação do artefato: a completa avaliação do artefato é de suma importância para a pesquisa, considerando-se que o artefato se relaciona a um problema específico;

- Contribuições da pesquisa: o artefato deve ser inovador, resolver um problema não resolvido, ou um problema conhecido de maneira mais eficaz;

- Rigor da pesquisa: o artefato deve ser rigorosamente definido, formalmente representado, coerente e internamente consistente;

- Design como um processo de pesquisa: o artefato inclui um processo de busca que constitui um espaço sobre o qual se constrói um problema e se implementa uma solução;

- Comunicação da pesquisa: os resultados da pesquisa em DS devem ser comunicados de forma eficaz, tanto para o público técnico como gerencial. 


\section{Análise dos estudos de caso}

O estudo de caso representa uma oportunidade para aplicar e analisar os benefícios do modelo proposto e para melhorar a compreensão da influência das incertezas nos processos de tomada de decisão.

A coleta de dados, realizada nos últimos três anos, foi feita a partir de dois casos da Embraer. Para melhorar a compreensão dos aspectos relevantes na definição de portfólios tecnológicos, a estratégia para o desenvolvimento de tecnologias no setor aeroespacial foi detalhada por meio de análise de documentos, pesquisas e entrevistas. Nesta fase da pesquisa, os projetos analisados foram: "Fly-by-wire" (FBW), retrospectivo, e carro voador ("Sky"), prospectivo.

No desenvolvimento do sistema FBW não houve planejamento de cenários como parte da estratégia tecnológica da organização, em função da falta de valor percebido pela liderança e equipes. Os alertas antecipados não foram percebidos, até que uma demanda de mercado impôs uma necessidade importante e mandatória, que poderia comprometer a sustentabilidade da organização. Experiências anteriores, como o projeto FBW, entre outros, trouxe um aprendizado para a organização em relação à importância do desenvolvimento estratégico baseado em cenários. Muitos profissionais fizeram treinamentos específicos e passaram a aplicar o método. A partir daí, algumas estratégias foram geradas a partir do uso de cenários.

Primeiramente, foi feita a análise de documentação interna (Embraer) e artigos científicos, para uma conscientização mais ampla desses desenvolvimentos. Depois, alguns profissionais chave, que atuaram ou atuam diretamente no desenvolvimento destas tecnologias, foram entrevistados. As entrevistas foram conduzidas por meio de um roteiro e registradas durante as entrevistas.

Quando necessário, esclarecimentos adicionais foram solicitados aos entrevistados. Outros profissionais, que também atuam nesses desenvolvimentos, foram convidados a responder uma pesquisa, elaborada no "Google Forms", com o objetivo de mitigar vieses e incompletudes.

Este estudo, que é parte de uma tese de doutorado, continua por meio da exploração de outros desenvolvimentos tecnológicos. Nesta fase, compartilhada neste artigo, foram explorados os seguintes tópicos: (1) incertezas e propriedades emergentes, e (2) mapa de escolhas críticas de capacidades dinâmicas.

\section{Incertezas e propriedades emergentes}

A análise das incertezas e propriedades emergentes busca explorar: a influência das incertezas no processo de tomada de decisão relacionado à base tecnológica da 
organização, os critérios de decisão considerados na evolução dos domínios tecnológicos (domínio de integração para domínio proprietário) e impactos das decisões sobre a organização, e ainda como as propriedades emergentes das tecnologias influenciaram a aprendizagem organizacional.

\section{Fly by Wire (FBW)}

O FBW é um sistema que controla as superfícies de comando de voo por meio de software embarcado (Spitzer, 2011). Segundo Niedermeier e Lambregts (2012), a substituição dos controles mecânicos por conexões elétricas pela Airbus no final da década de 1980, tornou o uso do FBW essencial para as fabricantes de aeronaves. Neste contexto, a tecnologia se tornou um fator chave para a primeira geração da família EMBRAER 170/190 (E1). A organização aceitou os riscos e incertezas quando decidiu incluir o FBW sem ter controle total sobre a tecnologia. A Embraer dominava o comportamento dinâmico da aeronave (TRL 5/6), mas não tinha a prontidão necessária para o desenvolvimento do FBW. Para garantir sua competitividade, decidiu transformar o domínio de integração da tecnologia em domínio proprietário (Chagas Jr., Leite, \& Jesus, 2016).

As incertezas tiveram um grande impacto nos processos de tomada de decisão. No desenvolvimento do FBW, um fator que influenciou a evolução do domínio de integração para domínio proprietário foi a experiência no desenvolvimento de produtos, em que o domínio de integração comprometeu a finalização do programa. A falta de conhecimento deixou a organização inteiramente dependente do fornecedor, que encontrou dificuldades técnicas na complexidade do sistema, comprometendo os prazos do programa. Este fato impediu o monitoramento adequado do desenvolvimento, dificultando a definição de soluções tecnológicas. Outro ponto relevante se refere à oportunidade de verticalizar o desenvolvimento da tecnologia, considerando as altas margens de retorno do investimento. O domínio proprietário aumentou o valor agregado do produto, reduziu o ciclo de desenvolvimento, proporcionou maior controle do ciclo de desenvolvimento, reduziu a dependência de fornecedores e permitiu a definição das melhorias tecnológicas a serem implementadas, favorecendo a retenção da propriedade intelectual.

As propriedades emergentes do sistema eram previsíveis, mitigando fatores negativos e potencializando os positivos. A aprendizagem organizacional evoluiu significativamente devido à formação de um grupo de especialistas e a base de conhecimento interna. Esses fatores aumentaram o poder de decisão interna, diminuíram a dependência dos fornecedores, introduziram melhorias nos processos, 
elevaram o nível de competitividade dos produtos e estabeleceram uma base tecnológica própria.

Os principais benefícios desta decisão foram independência tecnológica, processos mais robustos e maior qualidade do produto. Esses resultados também foram positivos para a maturidade da segunda geração de aeronaves Embraer 170/190 (E2) e KC390, incentivando a organização a expandir a iniciativa para um número maior de sistemas.

Quando a Embraer decidiu aderir ao sistema FBW, a intenção era obter domínio no desenvolvimento do sistema de comando de voo no nível de software. No nível do sistema, haveria aprendizado de todas as funções desse tipo, como controle de três eixos e proteção de envelope de voo - aprovados de acordo com a norma SAE ARP4754 (1996). No nível de software, a missão era ser capaz de desenvolver um software crítico de nível A pela norma RTCA DO-178B (1992). A decisão pelo software embarcado e integração de sistemas foi estratégica, e o desenvolvimento do software a partir da modelagem da arquitetura e componentes do sistema FBW evitou muitas negociações com fornecedores de sistemas, conferindo à organização autonomia e rapidez no ciclo de desenvolvimento.

O sistema de controle de voo é crítico por estar relacionado a aspectos de segurança, desempenho e eficiência da aeronave. É importante destacar a preservação da propriedade intelectual sobre as leis de controle, autonomia de decisão sobre mudanças, menores custos e ciclos de desenvolvimento, maior qualidade de software e integração de sistemas. A atividade de pesquisa começou sob demanda e após atingir a maturidade, passou a ter autonomia e gerar soluções para a área do programa.

\section{Carro voador (Projeto Sky)}

O comprometimento da infraestrutura de mobilidade urbana com o crescimento da população nos últimos anos está transformando os carros voadores em realidade. E as fabricantes de aeronaves perceberam uma grande oportunidade nessa tendência, tanto no modelo de negócios quanto no desenvolvimento e aceleração de novas tecnologias.

A busca por soluções que combinam o melhor do transporte terrestre com o transporte aéreo é um desafio. Uma solução possível é criar um sistema que busque superar os custos associados aos sistemas de transporte convencionais. As vantagens estão em minimizar o uso de infraestruturas rodoviárias, a liberdade pessoal de voar e a otimização do tráfego (Bülthoff, 2017, Ben- Haim, Ben- Haim, \& Shiftan, 2018).

Os carros voadores estão exigindo desenvolvimentos acelerados em um ambiente de incertezas comerciais e tecnológicas significativas que exigem decisões 
precoces, impactando os processos de tomada de decisão. Devido à demanda do mercado, a organização busca um modelo viável no desenvolvimento do carro voador, que envolve tecnologias maduras e tecnologias críticas de baixa maturidade, como sistemas autônomos e baterias elétricas. O tempo para amadurecimento e integração das tecnologias também é um fator de incerteza e requer múltiplas parcerias estratégicas.

Os ciclos de desenvolvimento tecnológico e de produtos são acelerados, e toda a cadeira precisa andar no mesmo ritmo. Desta forma, a Embraer não possui controle sobre esses ciclos e depende de conhecimento crítico dos parceiros para decisões importantes em relação às definições do portfólio de tecnologias.

As propriedades emergentes incluem flexibilidade de modelo, produto e negócio. Ao tornar o modelo mais flexível, o sistema pode gerar soluções alternativas. Essas propriedades, que impactaram significativamente os processos de desenvolvimento tecnológico da Embraer, envolvem a capacidade de capturar as propriedades no mercado e na sociedade, a capacidade de distinguir as propriedades críticas e a velocidade de reação da organização.

Os maiores benefícios desse desenvolvimento conjunto contemplam o rápido amadurecimento de tecnologias críticas. O conhecimento precisa se adaptar ao novo mercado e às informações dos usuários, estreitando as fronteiras entre os tomadores de decisões que podem se beneficiar das informações compartilhadas nesse consórcio de desenvolvimento. As principais características estão no novo modelo de transporte e negócios como um todo, não apenas no desenvolvimento de tecnologias. O projeto do carro voador segue uma tendência de mercado e a medição do sistema contempla ganhos tecnológicos, como autonomia e características associadas.

\section{Mapa de escolhas críticas de capacidades}

Esses casos também foram analisados em relação aos quadrantes definidos por Pisano (2016) em relação ao mapa das escolhas críticas para o desenvolvimento de capacidades (Figura 4).

As estratégias tecnológicas, com diferentes graus de incerteza, podem exigir investimentos em combinações específicas de capacidades e investimentos. Os casos analisados mostraram que a evolução das capacidades não foi aleatória e apenas uma parte dessa composição evoluiu naturalmente, devido a um processo histórico de desenvolvimento da organização. 
Figura 4: Mapa de escolhas críticas de capacidades

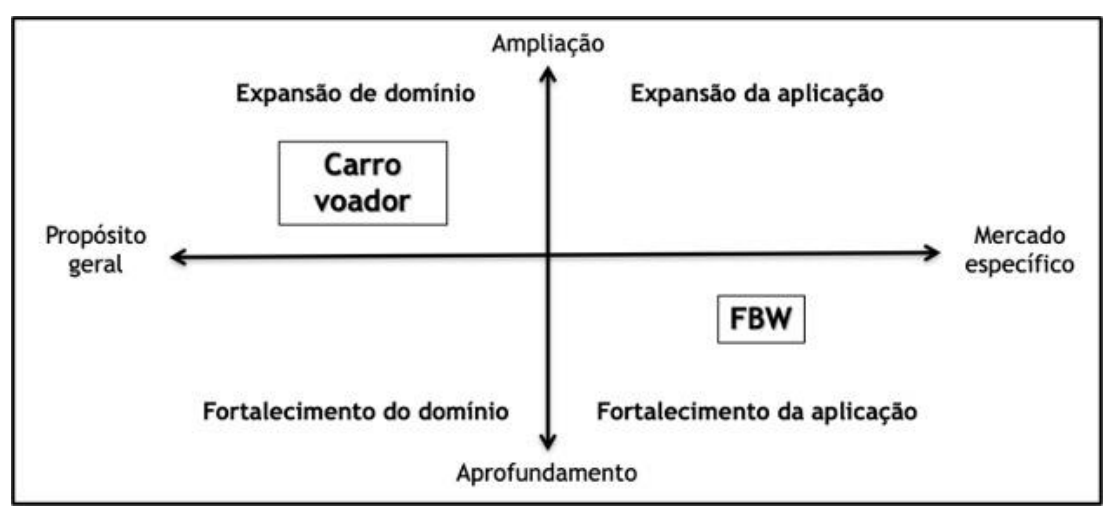

Fonte: adaptado de Pisano (2016)

\section{Fortalecimento da aplicação (Caso FBW)}

A Embraer, durante o desenvolvimento do E1, era capaz de integrar os sistemas FBW, mas não o seu crescimento vertical, que exigia o desenvolvimento de ferramentas internas específicas. Neste caso, o aprofundamento das capacidades está relacionado ao processo de melhoria do produto e as capacidades de ampliação às demandas do mercado por inovação. Para comandos de voo e tecnologias de certificação de produtos aeronáuticos, os recursos devem ser específicos, mas, para software embarcado, de propósito geral.

A concorrência na indústria tem impulsionado o desenvolvimento de capacidades para atender um objetivo claro, definindo uma distribuição de recursos equilibrada com tendência ao aprofundamento das capacidades em mercado específico. No entanto, uma análise estratégica mais relevante trouxe maior compreensão de como a nova capacidade alavancaria a competência tecnológica para o desenvolvimento do novo produto.

Além da viabilidade econômica, considerou-se a redução de riscos, melhoria dos ciclos de desenvolvimento e proteção da propriedade intelectual. A viabilidade econômica foi apenas uma segunda variável na equação de decisão, com foco na redução de custos associada à falta de maturidade dos sistemas que dependiam de fornecedores. Os sistemas eram seguros, mas imaturos, o que poderia levar a problemas operacionais.

A Embraer percebeu a necessidade de revisar a estratégia em relação às suas capacidades com base no risco que um fabricante de aeronaves assume com sistemas críticos e complexos, destacando a importância do domínio proprietário. Sem essa mudança estratégica, a organização dependeria de fornecedores, comprometeria a propriedade intelectual e teria o custo de não-maturidade de fornecedores de sistemas críticos. 


\section{Expansão de domínio (Caso carro voador - Projeto Sky)}

No projeto Sky, as incertezas são muito grandes, tanto do lado do cliente quanto da organização, que investiu no aumento das capacidades de propósito geral e estabeleceu as parcerias necessárias para capacidades específicas. Essa combinação estratégica está fortemente associada ao tempo necessário de amadurecimento da tecnologia e do programa.

A ampliação da capacidade considera o carro voador como um veículo diferente de uma aeronave convencional, definindo a busca pela ampliação da capacidade de propósito geral, por poder ser usada em outros desenvolvimentos. A Embraer possui o domínio proprietário de tecnologias aeronáuticas, mas deve participar do desenvolvimento de tecnologias ainda imaturas. Esse modelo estratégico que considera a combinação de diferentes capacidades visa acelerar o desenvolvimento de tecnologias de baixa maturidade para integração em um sistema de mobilidade igualmente imaturo, mas com estimativa de tempo de lançamento já definida (Leite \& Chagas Jr., 2019).

O projeto "Sky" representa um sistema altamente complexo, inovador, regulado e intensivo em tecnologias críticas e imaturas. O projeto está inserido em um ambiente competitivo e de incertezas. O projeto inclui a ampliação das capacidades de propósito geral (expansão do domínio), que moldam as futuras opções de entrada em novos mercados, embora seja difícil prever o lado da demanda (Leite \& Chagas Jr., 2019).

\section{Modelo proposto (artefato) e verificação das diretrizes DS}

\section{Modelo de desenvolvimento dinâmico e adaptativo}

O mercado atual, caracterizado por múltiplas incertezas e mudanças exige mais agilidade das organizações. Desta maneira, a busca pelo melhor desempenho no modelo de respostas e definições estratégicas pode impactar a flexibilidade das organizações, por estarem diretamente relacionadas ao comportamento de seus tomadores de decisão.

À medida que o futuro evolui, os tomadores de decisão procuram responder às novas situações adaptando seus planos à nova realidade (McInerney, Lempert, \& Keller, 2012). Essa adaptação de um projeto ao longo do tempo não deve ser determinada apenas pelo que é conhecido, mas pela construção de um conhecimento. A partir dessa situação, Hallegatte, Shah, Lempert, Brown e Gill (2012) abordam o conceito de incerteza, que demanda planos adaptativos e dinâmicos. Esses planos envolvem ações de curto prazo, ao mesmo tempo em que estabelecem uma estrutura para o direcionamento de atividades futuras (Albrechts, 2004). Segundo Haasnoot, 
Kwakkel, Walker e Ter Maat (2013), o tomador de decisão deve criar uma visão estratégica de futuro, mantendo seu compromisso com as ações de curto prazo. Um modelo de desenvolvimento que incorpora esses fatores permite uma adaptação dinâmica ao longo do tempo.

O modelo é baseado no ambiente que caracteriza as organizações do setor aeroespacial. Cada vez mais, as soluções para problemas complexos dependem da capacidade da organização combinar suas visões estratégicas (futuras) com ações de curto prazo, bem como a tomada de decisão sob incerteza. Esses fatores exigem modelos dinâmicos que respondam rapidamente às mudanças impostas pelo mercado e às necessidades identificadas. Busca-se, com o modelo de desenvolvimento dinâmico e adaptativo, melhorar o desempenho das organizações em relação às suas estratégias tecnológicas (Figura 5).

Figura 5: Modelo dinâmico adaptativo para desenvolvimento de estratégias tecnológicas

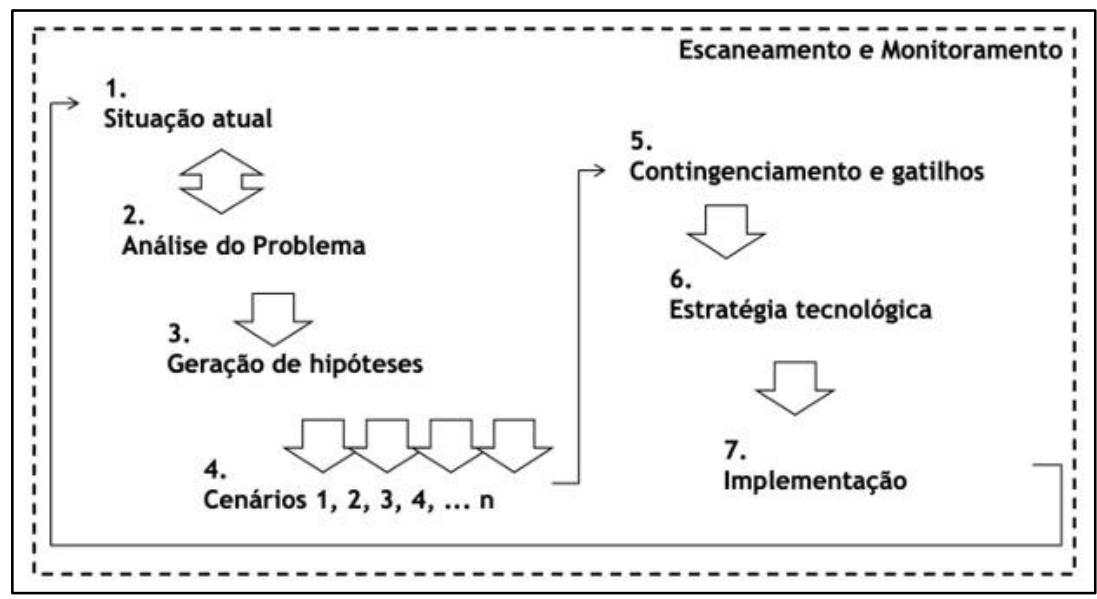

Fonte: Leite \& Chagas Jr., 2019

A etapa 1 contempla a análise da situação atual, interna e externa à organização, que inclui as principais expectativas, tendências e incertezas. A etapa 2 se concentra na análise de problemas, oportunidades e vulnerabilidades das tecnologias, produtos, negócios e regulamentações.

Nesta fase, os níveis de TRL devem ser considerados por influenciar diretamente as definições estratégicas, especialmente em termos do tempo necessário para o desenvolvimento da tecnologia aeroespacial e investimentos (Figura 6). 
Figura 6: Custo e Motivação para Investimento em Desenvolvimento Tecnológico

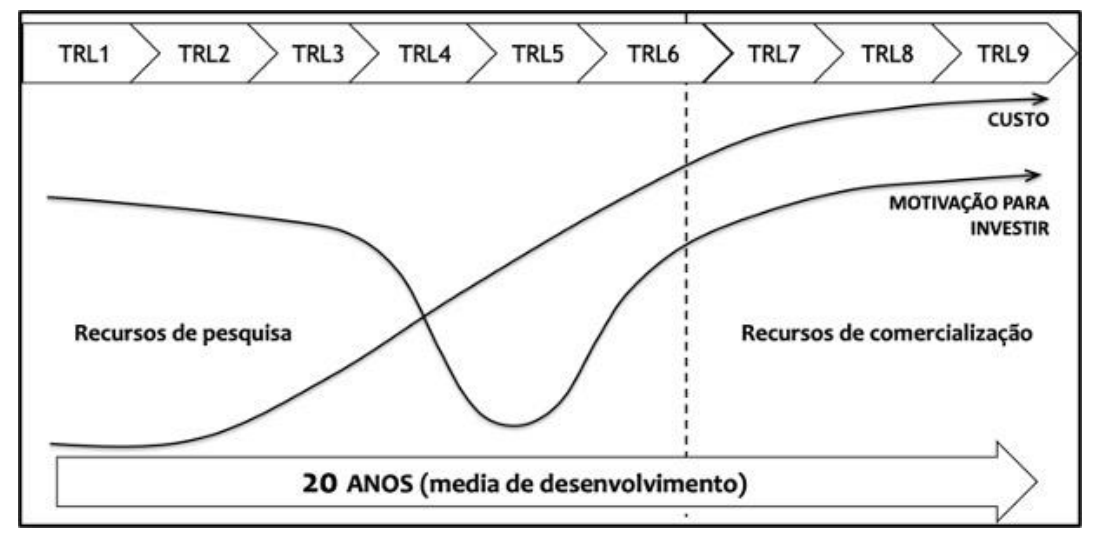

Fonte: Leite \& Chagas Jr., 2019

Nesta fase, os níveis de TRL devem ser considerados pela forte influência nas definições estratégicas, especialmente em termos do tempo necessário para o desenvolvimento da tecnologia aeroespacial e investimentos (Figura 6).

O custo do desenvolvimento de tecnologia cresce continuamente, mas o comportamento da motivação do investimento é diferente. Por um lado, o interesse em pesquisa e do outro lado, mercado, a motivação para o desenvolvimento de produtos. Entre esses extremos, há um período crítico de transição em que muitos eventos são interrompidos pela falta de recursos para o desenvolvimento da tecnologia até o estágio em que as organizações podem explorar seu potencial comercial. A redução desse período de transição, possível por meio da melhor integração entre as estratégias "technology push" e "demand pull", pode melhorar a taxa de sucesso de tecnologias que atendem às necessidades do mercado.

O objetivo da etapa 3 é a geração de hipóteses, com diferentes perspectivas. Nesse ponto, o conceito do sistema começa a ser definido, mesmo sem informações suficientes. E na etapa 4, a construção do cenário permite uma exploração sistêmica de alternativas futuras. As etapas 3 e 4 estão representadas na Figura 7.

Na etapa 5, a informação a ser monitorada deve ser definida por incluir os fatores que dinamizam o modelo. A etapa 6 define a estratégia tecnológica com base nos resultados das ações anteriores, com o desafio de manter as opções abertas durante a maior parte do tempo. Na etapa 7, as ações estratégicas são implementadas e priorizadas nos horizontes de curto, médio e longo prazo, permitindo definir o que manter, o que atrasar e o que descontinuar. 
Figura 7: Construção de cenários e Geração de Hipóteses

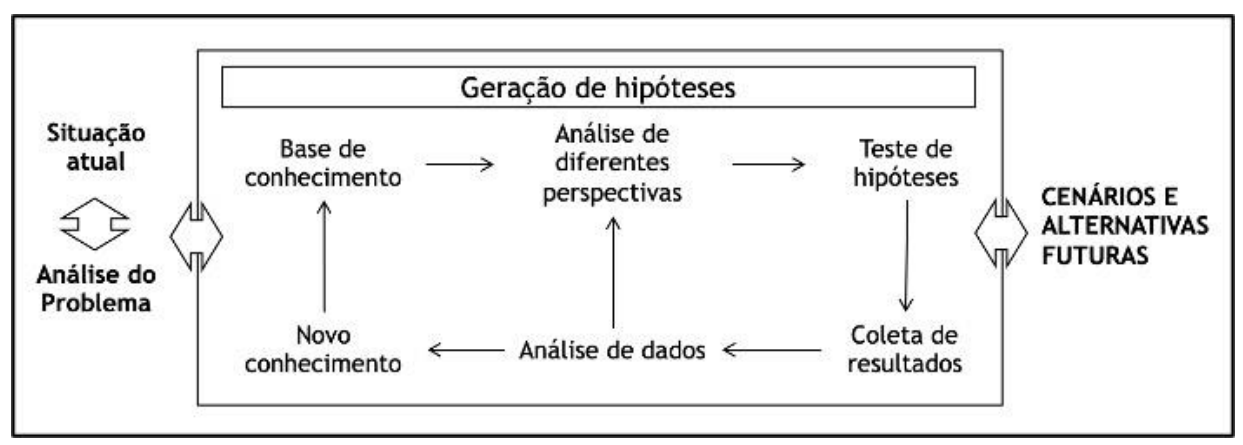

Fonte: Leite \& Chagas Jr., 2019

Ao longo da aplicação do modelo, as informações devem ser monitoradas, analisadas e relacionadas aos gatilhos. Assim, as ações estratégicas são iniciadas, alteradas, interrompidas ou expandidas em resposta aos dados, aumentando a flexibilidade do sistema.

\section{Verificação das diretrizes DS}

Este capítulo detalha o atendimento às diretrizes do DS para o artefato, segundo Hevner, March, Park e Ram (2004).

- Diretriz 1: Neste estudo, o artefato corresponde ao modelo de desenvolvimento dinâmico adaptativo para a estratégia tecnológica no setor aeroespacial.

- Diretriz 2: A seleção de tecnologias contempla os critérios a serem considerados no desenvolvimento das tecnologias e produtos conceito em longo prazo, capacitação de fornecedores e formação de capacidades dinâmicas. Estes fatores, contemplados no artefato proposto, buscam melhorar geração de oportunidades e a capacidade de tomada de decisões.

- Diretriz 3: A avaliação do artefato inclui uma visão prospectiva no setor aeroespacial, com elementos que buscam assegurar flexibilidade e dinamismo à seleção de tecnologias.

- Diretriz 4: O artefato ajuda os tomadores de decisão a desenvolverem estratégias de adaptação para a seleção de tecnologias no setor aeroespacial, considerando oportunidades, ameaças e opções de desenvolvimento identificadas. As estratégias devem ser flexíveis para se adaptarem às condições de incertezas e aproveitarem melhor as oportunidades.

- Diretriz 5: O artefato proposto resulta da evolução de modelos adaptativos por meio da análise de estudos de casos reais em projetos retrospectivo e prospectivo no setor aeroespacial.

- Diretriz 6: O desenvolvimento das pesquisas inclui análise de dados, pesquisa e entrevistas, para melhor compreensão dos casos selecionados. 
- Diretriz 7: Resultados parciais deste estudo foram apresentados em eventos nacionais e internacionais.

\section{Conclusão}

A pesquisa se desenvolveu a partir da hipótese de que as organizações complexas e inseridas em ambientes de incertezas não possuem uma gestão estratégia eficaz para detectar e aproveitar oportunidades. Neste contexto, propõe-se um modelo que tem o objetivo de agilizar a identificação, interpretação e resposta às mudanças do ambiente, melhorando os resultados da gestão estratégica de organizações do setor aeroespacial.

Para responderem às mudanças descontínuas, as organizações precisam de uma estrutura mais integrada, dinâmica e que possa se adaptar continuamente às demandas internas e externas. A integração na captura de oportunidades e nas tomadas de decisão também foi uma lacuna identificada.

A fim de melhorar estes fatores, o estudo buscou a expansão do conceito "generative sensing" para "generative sensing e seizing", em um modelo estratégico que busca (1) melhorar a captura e o aproveitamento de oportunidades, (2) melhorar a vantagem competitiva da organização e (3) contribuir com uma estratégia tecnológica mais robusta em um horizonte de longo prazo.

Grandes oportunidades podem surgir em ambientes incertos, exigindo mudanças organizacionais rápidas. Desta forma, os benefícios da agilidade estratégica aumentam com o nível de incerteza inerente aos ambientes de P\&D. Nesse ambiente, as transformações tecnológicas exigem estratégias mais flexíveis.

O modelo de desenvolvimento dinâmico adaptativo busca alinhar as capacidades dinâmicas aos futuros alternativos, gerados pelos cenários prospectivos, buscando dar mais agilidade e flexibilidade ao desenvolvimento estratégico. A proposta foi desenvolvida através de estudo de casos com o objetivo de melhorar as tomadas de decisão em condições de incertezas.

O modelo envolve a coleta de informações, modelagem e simulação, e o monitoramento que permeia todo o processo. A primeira parte permite ao tomador de decisão entender o ambiente e definir os fatores de atenção ou mudanças estratégicas. Na segunda parte, o entendimento das múltiplas dimensões do ambiente (hipóteses) é estruturado com o desenvolvimento de cenários, que permitem a modelagem e simulação de informações (lógica abdutiva), melhorando o nível de confiança da tomada de decisão em condições de incerteza. O monitoramento contínuo das informações favorece a detecção de condições críticas que podem indicar potenciais gatilhos de mudança. 
Ao influenciar a flexibilidade estratégica da organização, o modelo favorece a aprendizagem organizacional e a criação de soluções adaptativas. Daí a importância de repetir o processo ao longo da evolução dos diferentes níveis de maturidade tecnológica (TRL).

Os casos explorados nesta pesquisa permitiram o detalhamento dos impactos das tomadas de decisão sobre o desenvolvimento, a natureza e o domínio tecnológico na definição das rotas tecnológicas e fatores associados à estratégia organizacional. A pesquisa destacou, como principais deficiências da estratégia tecnológica, as tomadas de decisão sob incerteza, a falta de integração entre as áreas nas tomadas de decisão e a amplitude e horizonte de tempo da estratégia, fatores que impactam diretamente a flexibilidade estratégica da organização.

\section{Referências}

Albrechts, L. (2004). Strategic (spatial) planning reexamined. Environment and Planning $B, 31(5), 743-758$.

Augier, M., \& Teece, D. J. (2008). Strategy as Evolution with Design: The Foundations of Dynamic Capabilities and the Role of Managers in the Economic System. Organization Studies, 29(8-9), 1187-1208.

Azizian, N., Sarkani, S., \& Mazzuchi, T. (2009). A comprehensive review and analysis of maturity assessment approaches for improved decision support to achieve efficient defense acquisition. In Proceedings of the World Congress on Engineering and Computer Science, 2, San Francisco, CA.

Barney, J. (1991). Firm resources and sustained competitive advantage. Journal of Management, 17(1), 99-120.

Ben-Haim, R., Ben-Haim, G., \& Shiftan, Y. (2018). Penetration and impact of advanced car technologies. MOJ Civil Eng., 4 (4), 175-184.

Brown, S. L., \& Eisenhardt, K. (1997). The Art of Continuous Change: Linking Complexity Theory and Time-Paced Evolution in Relentlessly Shifting Organizations. Administrative Science Quarterly, 42(1), 1-34.

Bülthoff, H. (2017). Flying Air Taxis: the next big game changer? Talk presented at Universität UIm: Colloquium Cognitive Systems. Ulm, Germany.

Chagas Junior, M. F., Leite, D. E. S., \& Jesus, G. T. (2017). 'Coupled processes' as dynamic capabilities in systems integration. Revista de Administração de Empresas, 57(3), 245-257.

Courtney, H., Kirkland, J., \& Viguerie, P. (1997). Strategy Under Uncertainty. Harvad Business Review, 67-79.

De Sordi, J. O., Azevedo, M. C., Meireles, M. A., \& Campanario, M. A. (2013). A Abordagem Design Science no Brasil segundo as publicações em administração da informação. In: Encontro da Associação Nacional de Pós-Graduação e Pesquisa em Administração (EnANPAD), 2013, Rio de Janeiro. XXXVII Encontro da ANPAD, 1, 1-16. 
Dong, A., Garbuio, M., \& Lovallo, D. (2016). Generative sensing: a design perspectivemicro-foundationsndations of sensing capabilities. California Management Review, 58(4), 97-117.

Drucker, P. (2011). The age of discontinuity: guidelines to our changing society. Transaction Publishing, New Jersey.

Eisenhardt, K. M. (1989). Building theories from case study research. Academy of Management Review, 14(4), 532-550.

Eisenhardt, K. M., \& Martin, J. A. (2000) Dynamic capabilities: what are they? Strategic Management Journal, 21, 1105-1121.

Eisenhardt, K. M., \& Sull, D. (2001). Strategy as simple rules. Harvard Business Review, 79(1), 106-119.

Eisenhardt, K. M., Graebner, M. E. (2007). Theory building from cases: opportunities and challenges. Academy of Management Journal, 50(1), 25-32.

Grant, R. M. (1991). The Resource-Based Theory of Competitive Advantage: Implications for Strategy Formulation. Californian Management Review, 33(3), 114-135

Godet, M. (1983). Méthode des Scénarios. Revista Futuribles.

Haasnoot M., Kwakkel J. H., Walker, W., \& ter Maat, J. (2013). Dynamic adaptive policy pathways: a new method for crafting Robust decisions for a deeply uncertain world. Global Environmental Change, 23(2), 485-498.

Hallegatte, S., Shah, A., Lempert, R., Brown, C., \& Gill, S. (2012). Investment decision making under deep uncertainty application to climate change. The World Bank.

Helfat, C. E., \& Peteraf, M. A. (2009). Understanding dynamic capabilities: progress along a developmental path. Strategic Organization, 7(1), 91-102.

Hevner, A. R., March, S. T., Park, J., \& Ram, S. (2004). Design science in information systems research. MIS Quarterly, 28(1), 75-105.

Kwakkel, J. H., Pruyt, E. (2013). Exploratory Modeling and Analysis, an approach for model-based foresight under deep uncertainty. Technological Forecasting and Social Change, 80(3), 419-431.

Levinthal, D. (1992) Surviving Schumpeterian environments: an evolutionary perspective. Industrial Corporate Change, 1(3), 427-443.

Mankins, J. C. (2009a). Technology readiness and risk assessments: a new approach. Acta Astronautica, 65, 1208-1215.

Mankins, J. C. (2009b). Technology readiness assessment: a retrospective. Acta Astronautica, 65, 1216-1223.

Mankins, J. C. (2002). Approaches to strategic research and technology (R\&T) analysis and road mapping. Acta Astronautica, 51(1-9), 3-21.

McInerney, D., Lempert, R., \& Keller, K. (2012). What are robust strategies in the face of uncertain climate threshold responses. Climate Change, 112, 547-568. 
Niedermeier, D., \& Lambregts, A. A. (2012). Fly-by-Wire augmented manual control - basic design considerations, ICAS 2012, 28th International Congress of the Aeronautical Sciences (ICAS), Brisbane, Australia.

Peirce, C. S. (1998). The Essential Peirce. Selected Philosophical Writings, 2, 18931913. The Peirce Edition Project, (Eds.) Bloomington: Indiana University Press.

Peirce, C. S. (1974) Collected papers de Charles Sanders Peirce. In: Hartshorne, C., Weiss, P., Burks, A. (Eds.) Cambridge, Massachusetts, Harvard University Press, 1-8.

Peirce, C. S. (1958) The fixation of belief. In: Hartshorne, C., Weiss, P., Burks, A. (Eds.), Collected Papers of Charles Sanders Peirce, Cambridge, Massachusetts, Harvard University Press, 5, 223-247, 1931-1935 (First published 1877).

Penrose, E. T. (1959). The theory of the growth of the firm. New York: John Wiley.

Pisano, G. P. (2016). Toward a prescriptive theory of dynamic capabilities: connecting strategic choice, learning, and competition. Industrial and Corporate Change, 26(5), 747762.

Prahalad, C. K., \& Hamel, G. (1990). The core competence of the Corporation. Harvard Business Review, 79-91.

Ramírez, R., \& Selin, C. (2014). Plausibility and probability in scenario planning. Foresight, 16(1), 54-74.

Rohrbeck, R. (2010). Corporate foresight: towards a maturity model for the future orientation of a firm. Physica-Verlag, Springer, Heidelberg and New York.

Rohrbeck, R. \& Schwarz, J. O. (2013). The value contribution of strategic foresight: Insights from an empirical study of large European companies. Technological Forecasting and Social Change, Elsevier, 80(8), 1593-1606.

Rohrbeck, R. (2014). Trend Scanning, Scouting and Foresight Techniques. In Management of the Fuzzy Front End of Innovation; Gassmann, O., Schweitzer, F., Eds.; Springer: Cham, Switzerland, 59-73.

RTCA Inc. (1992). DO-178B software considerations in airborne systems and equipment certification, Washington, D.C., EUA.

SAE International. (1996). ARP-4754 certification considerations for highly integrated or complex aircraft systems, Warrendale, PA, EUA.

Schoemaker, P. J. H. (1995). Scenario planning: a tool for strategic thinking. Sloan Management Review, 36(25).

Schwartz, P. (1991). The Art of the Long View: Planning for the Future in an Uncertain World. Doubleday Currency, New York.

Schwarz, J. O. (2008). Assessing the future of future studies in management. Futures, 40(3), 237-246.

Schwarz, J. O., Rohrbeck, R., Wach, B. (2019). Corporate foresight as a microfoundation of dynamic capabilities. Future Foresight Sci., 2 (2), e28, 1-11.

Simon, H. A. (1996). The sciences of the artificial. 3rd. ed. Cambridge, MA: Massachusetts Institute of Technology Press. 
Spitzer, C. R. (2011). The Avionics Handbook. CRC Press.

Teece, D. J., \& Pisano, G. (1994). The dynamic capabilities of firms: an Introduction. Industrial and Corporate Change, 3(3), 537-556.

Teece, D. J., Pisano, G., \& Shuen, A. (1997). Dynamic capabilities and strategic management. Strategic Management Journal, 18(7), 509-533.

Teece, D. J. (2007). Explicating dynamic capabilities: the nature and microfoundations of (sustainable) enterprise performance. Strategic Management Journal, 28(13), 1319-1350.

Teece, D. J., Peteraf, M., \& Leih, S. (2016). Dynamic capabilities and organizational agility: risk, uncertainty, and strategy in the innovation economy. California Management Review, 58(4), 13-35.

Teece, D. J. (2018). Business models and dynamic capabilities, Long Range Planning, $51,40-49$.

Van der Heijden, K., Bradfield, R., Burt, G., Cairns, G. \& Wright, G. (2002). Sixth Sense: Accelerating Organisational Learning with Scenarios. John Wiley \& Sons. \& Sons.

Van der Heijden, K. (2005). Scenarios: The Art of Strategic Conversation. John Wiley

Wack, P. (1985). Scenarios: shooting the rapids. Harvard Business Review, 140-150.

Wernerfelt, B. (1984). A Resource-based View of the Firm. Strategic Management Journal, 5(2), 171-180.

Winter, S. G. (2004). Specialised perception, selection, and strategic surprise: learning from the Moths and Bees. Long Range Planning, 37(2), 163-169. 